\title{
ARTICLE Colitis susceptibility in mice with reactive oxygen species deficiency is mediated by mucus barrier and immune defense defects
}

Gabriella Aviello ${ }^{1,2,3,6}$, Ashish K. Singh ${ }^{1,2}$, Sharon $\mathrm{O}^{\prime}$ Neill $^{1,7}$, Emer Conroy ${ }^{1}$, William Gallagher ${ }^{1}$, Giuseppe D’Agostino ${ }^{4}$, Alan W. Walker ${ }^{3}$, Billy Bourke ${ }^{2}$, Dimitri Scholz ${ }^{5}$ and Ulla G. Knaus ${ }^{1,2}$

Reactive oxygen species (ROS) generated by NADPH oxidases (NOX/DUOX) provide antimicrobial defense, redox signaling, and gut barrier maintenance. Inactivating NOX variants are associated with comorbid intestinal inflammation in chronic granulomatous disease (CGD; NOX2) and pediatric inflammatory bowel disease (IBD; NOX1); however Nox-deficient mice do not reflect human disease susceptibility. Here we assessed if a hypomorphic patient-relevant CGD mutation will increase the risk for intestinal inflammation in mice. Cyba (p22 ${ }^{\text {phox }}$ ) mutant mice generated low intestinal ROS, while maintaining Nox4 function. The Cyba variant caused profound mucus layer disruption with bacterial penetration into crypts, dysbiosis, and a compromised innate immune response to invading microbes, leading to mortality. Approaches used in treatment-resistant CGD or pediatric IBD such as bone marrow transplantation or oral antibiotic treatment ameliorated or prevented disease in mice. The Cyba mutant mouse phenotype implicates loss of both mucus barrier and efficient innate immune defense in the pathogenesis of intestinal inflammation due to ROS deficiency, supporting a combined-hit model where a single disease variant compromises different cellular functions in interdependent compartments.

Mucosal Immunology (2019) 12:1316-1326; https://doi.org/10.1038/s41385-019-0205-x

\section{INTRODUCTION}

In the gastrointestinal (Gl) tract, the production of reactive oxygen species (ROS) by nicotinamide adenine dinucleotide phosphate (NADPH) oxidases (NOX/DUOX) is a key biological mechanism regulating pathogen killing, ${ }^{1}$ host-microbe interactions, ${ }^{2}$ and tissue repair after injury. ${ }^{3}$ Loss-of-function variants in one of the genes encoding for the NOX2 NADPH oxidase complex (CYBB, CYBA, NCF1, NCF2, NCF4) cause chronic granulomatous disease (CGD), a primary immunodeficiency characterized by recurrent bacterial or fungal infections and aberrant inflammation. Approximately $40-60 \%$ of CGD patients develop comorbid GI manifestations (CGD-IBD) including recurrent diarrhea, perianal disease, and rectal abscesses with combined features of Crohn's disease ${ }^{4,5}$ and ulcerative colitis. ${ }^{6,7}$ Mainly affected is the colon ${ }^{8}$ with thickened bowel wall, crypt abscesses, and mucosal cell infiltration. ${ }^{5,9}$ The development of IBD in CGD patients correlates with the presence of common IBD risk alleles, although risk burden is significant lower than in pediatric CD patients, indicating that defective NOX2 activity constitutes a major IBD risk factor. ${ }^{10}$ Optimal treatment of the Gl symptoms in CGD patients is often difficult as anti-TNF and immunosuppressive agents can predispose to infections.
However, compromised redox homeostasis has much wider implications in human intestinal health. Inherited and sporadic cases of pediatric inflammatory bowel disease (IBD) without an underlying primary immunodeficiency are increasingly connected to inactivating variants in several NADPH oxidase enzymes (NOX1, DUOX2) and overall decreased ROS levels in phagocytes. ${ }^{2,11}$ Improved understanding of intestinal inflammation due to attenuated ROS requires mechanistic insights in diseaserecapitulating animal models. This has been challenging in current mouse models of Nox/Duox deficiency, ${ }^{2}$ possibly due to compensation by related oxidase isoforms or other ROS sources, ${ }^{12}$ as $\mathrm{H}_{2} \mathrm{O}_{2}$ is relatively stable and diffusible. Further, environmental effects present in humans including diet or exposure to pathogens are minimized in the controlled setting prevalent in animal research.

To identify processes triggering intestinal inflammation in low ROS conditions, we systematically studied multiple mouse strains with Nox isoform deficiency or inactivation in experimental colitis models while maintaining constant health and environmental conditions. Here we report that mice with Nox1, Cybb, or Cyba deletion do not display increased susceptibility to colitis, while

\footnotetext{
${ }^{1}$ Conway Institute, University College Dublin, Dublin, Ireland; ${ }^{2}$ National Children's Research Center, Our Lady's Children's Hospital Crumlin, Dublin, Ireland; ${ }^{3}$ The Rowett Institute,

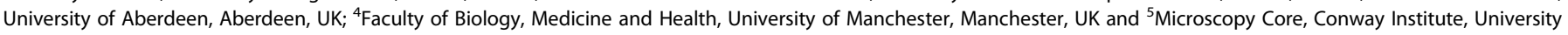
College Dublin, Dublin, Ireland

Correspondence: Ulla G. Knaus (ulla.knaus@ucd.ie)

${ }^{6}$ Present address: Department of Pharmacy, University of Naples Federico II, Naples, Italy

${ }^{7}$ Present address: Legend Biotech Ireland Ltd, Dublin, Ireland

This study is dedicated to the memory of our friend and colleague Malgorzata Kubica.
}

Received: 2 July 2019 Revised: 13 August 2019 Accepted: 6 September 2019

Published online: 25 September 2019 
mice with a Cyba missense mutation are prone to severe colon inflammation due to their underlying gut barrier and innate immunity defects. This Cyba mutation leads to an altered redox state in the intestine that cannot be compensated and represents a combined-hit model that impacts ROS-associated mucosal and innate defense mechanisms via a single gene mutation.

\section{RESULTS}

The Cyba mutation renders mice highly susceptible to severe colitis

CGD patients have impaired phagocyte function causing exuberant inflammatory responses to microbes with transmural immune cell infiltration in the colon. These features can be recapitulated in mice by using colitis models, such as (i) the DSS model, responsive to mucus-associated alterations, microbiota and innate immune defects, (ii) an acute TNBS model, driven by $\mathrm{T}$ lymphocyte responses and accompanied by abundant neutrophil influx, and (iii) an acute bacterial infection model which is influenced by microbiota-triggered colonization resistance in the context of epithelial ROS. ${ }^{12}$

We used homozygous Cyba $a^{n m f 33}$ mice harboring a missense mutation in $\mathrm{p} 22^{\text {phox }}$ leading to a tyrosine to histidine amino acid substitution $(\mathrm{Y} 121 \mathrm{H})$, which prevents superoxide generation in neutrophils (Nox2) and causes vestibular defects (Nox3). ${ }^{13}$ When treated with DSS, Cyba $a^{\text {nmf333 }}$ mice developed severe colitis with rapid body weight loss and increased disease scores when compared with wild-type (WT) mice (Fig. 1a, b). Further examination of $C y b a^{\text {nmf333 }}$ mice revealed shorter colon length (Fig. 1c) and transmural inflammation characterized by increased cell infiltration in the mucosa, complete loss of crypt architecture, and submucosal edema (Fig. $1 \mathrm{~d}$, e). The severity of disease led to high mortality, as all Cyba $a^{n m 333}$ mice reached the humane endpoint between day 7 and 8 (Fig. 1f). At the disease peak Cyba $a^{n m f 333}$ mice exhibited hypothermia (Fig. 1g), increased expression of colonic antimicrobial peptide Reg3y (Fig. 1h), and bacterial translocation (Fig. 1i), suggesting that the mortality occurred as consequence of a sepsis-like condition. Notably, the severe disease phenotype of $C y b a^{n m f 333}$ mice was also observed in heterozygous male Cyba $a^{+/ n m f 333}$ mice (Fig. S1a-e), while female heterozygous mice presented with a milder in vivo outcome (Fig. S1f-j). Similar susceptibility to colitis was also observed using the pre-sensitized acute TNBS model (Fig. 1j). While WT mice spontaneously recovered starting from day 2, Cyba $a^{\text {nmf333 }}$ mice continued to lose body weight and reached $50 \%$ mortality by day 4 (Fig. $1 \mathrm{k}, \mathrm{l}$ ), presumably due to attenuated tissue restitution. ${ }^{14}$ Inflammation and tissue damage were significantly increased in $C y b a^{n m f 333}$ mice on day 4 as shown by a higher colon weight/ length ratio and elevated pathology scores (Fig. 1m, n).

We also used the Citrobacter rodentium colitis model (Fig. S2a), which depends on colonization resistance, nutrient availability, and aerobic respiration. ${ }^{15,16}$ Bacterial colonization and histology in cecum and colon were comparable between $C y b a^{n m f 333}$ and WT mice (Fig. S2b-g). This result contrasts with global Nox1-4 inactivation in $\mathrm{Cyba}^{-/-}$mice, which were protected from $C$. rodentium infection due to overgrowth of beneficial Lactobacillus species. ${ }^{12}$ Steady-state intestinal permeability, Gl transit time, fecal IgA levels, and antimicrobial peptides $L y z 1$, Ang4, and Reg3g were all comparable between WT and Cyba ${ }^{\text {nmf333 }}$ mice (Fig. S3a-f), suggesting that other factors underlie the severe response of Cyba $a^{n m f 33}$ mice to colitogenic challenges.

Single Nox isoform deletion or global Nox inactivation do not exacerbate acute colitis

Cyba encodes for $\mathrm{p} 22^{\text {phox }}$, a partner protein required for Nox1-4 activity. ${ }^{17}$ To investigate whether colitis susceptibility in Cyba ${ }^{\text {nmf333 }}$ mice was caused by inactivation of Nox enzymes in the intestinal epithelium (Nox1) or the innate immune compartment (Nox2) DSS colitis studies in mice deficient in these Nox isoforms were performed (Fig. S4a). Mice with deletion of Nox2 $\left(C y b b^{-/-}\right)$ showed reduced signs of colitis (Fig. S4b, g), indicating that loss of Nox2 in mice is, in contrast to CGD patients with loss-of-function $C Y B B$ variants, protective with decreased weight loss or tissue injury. Disease progression and recovery in Nox $1^{-1-}$ mice was comparable to WT mice (Fig. S4b-e), although Nox $1^{-1-}$ mice showed reduced mucosal damage and inflammatory cell infiltration (Fig. S4f, g) as observed by others. ${ }^{2}$ The TNBS colitis phenotype of $\mathrm{Nox}^{-1-}$ and $\mathrm{Cybb}^{-/-}$mice was comparable to WT mice in the acute phase, but exacerbated in the restitution phase due to compromised wound healing. ${ }^{2}$ To broaden our approach, we induced colitis in $\mathrm{Cyba}^{-1-}$ mice with global Nox1-4 inactivation due to loss of $\mathrm{p} 22^{\text {phox }}$. Cyba ${ }^{-1-}$ mice showed body weight loss, disease scores, and mortality rates comparable to matched WT mice (Fig. S4h-j) and did not develop enhanced colon pathology (Fig. S4k-m), probably due to compensatory epithelial barrier changes mediated by $\mathrm{H}_{2} \mathrm{O}_{2}$-producing lactobacilli. ${ }^{2,12}$ These data demonstrate that selective or global Nox deficiency in mice does not recapitulate the severe colitis phenotype observed in Cyba $a^{\text {nmf333 }}$ mice.

Characterization of the redox state in $C y b a^{\text {nmf333 intestine }}$ We set out to determine the molecular and physiological mechanisms for the apparent differences in colitis susceptibility of $C y b a^{\text {nmf333 }}$ vs Cyba $a^{-1-}$ mice. Earlier we reported that the Cyba $a^{\text {nmf333 }}$ mutation ( $\left.22^{\text {phox }} \mathrm{Y} 121 \mathrm{H}\right)$, inhibits complex formation, maturation, cell surface localization, and catalytic activity of NOX1 and NOX2, while assembly and functionality of the NOX4-p22 $22^{\text {phox }}$ Y121H complex was not affected. ${ }^{18,19}$ Three amino acids upstream of Tyr121 is a mutational hotspot in human CYBA p.Ser118Arg (Fig. 2a), resulting in CGD in at least eight patients (http:// structure.bmc.lu.se/idbase/CYBAbase/). NOX4-p22 ${ }^{\text {phox }}$ complex modeling suggested that substitution of Ser118 with arginine may convey a similar selectivity in facilitating NOX4-p22 $22^{\text {phox }}$ heterodimerization as observed for the Tyr121 to histidine change. $^{19}$ Transient expression of NOX4 with $\mathrm{p}_{22^{\text {phox }}}$ WT or mutants in NOX/DUOX/p22 $2^{\text {phox }}$ deficient epithelial cells resulted in comparable $\mathrm{H}_{2} \mathrm{O}_{2}$ generation, protein expression, and cell surface localization (Fig. $2 \mathrm{~b}-\mathrm{d}$ ), indicating that $\mathrm{Cyb} a^{n m f 333}$ mice functionally resemble CGD patients with the CYBA p.Ser118Arg variant, both retaining Nox4 activity.

In vivo chemiluminescence imaging was used to quantify the physiological ROS levels in the intestine of untreated Noxdeficient/inactivated mice. WT and $\mathrm{Cybb}^{-/-}$(Nox2-deficient) mice produced a similar ROS signal, while the signal was strongly reduced in $\mathrm{Nox}^{-1-}$ mice (Fig. 2e, f). This indicates that Nox2derived superoxide generated by innate immune cells does not contribute to the intestinal ROS signal at steady state and suggests that mainly epithelium-produced superoxide (Nox1) regulates barrier dynamics in homeostasis. The low ROS signal present in $\mathrm{Nox}^{-1-}$ mice further decreased in Cyba ${ }^{\text {nmf333 }}$ mice, while no signal was detected in $\mathrm{Cyba}^{-1-}$ mice (Fig. 2e-g). These results suggest that while Nox 1 provides the majority of intestinal epithelial ROS detectable by L-012 chemiluminescence, another Nox isoform inhibited by the $\mathrm{p} 22^{\text {phox }} \mathrm{Y} 121 \mathrm{H}$ mutation, possibly Nox $3{ }^{13}$ may contribute to barrier ROS. $\mathrm{H}_{2} \mathrm{O}_{2}$ production is not detected by the L-012 chemiluminescent probe in vivo, as no signal remained in $\mathrm{Cyba}^{-1-}$ mice, although $\mathrm{H}_{2} \mathrm{O}_{2}$-generating Nox4 and Duox 2 oxidases are not affected by $\mathrm{p} 22^{\text {phox }} \mathrm{Y} 121 \mathrm{H} .^{18}$ Nox4, which is difficult to detect in the intestine with existing antibodies, heterodimerizes with $\mathrm{p} 22^{\text {phox }} \mathrm{Y} 121 \mathrm{H}$, which will enhance $\mathrm{p} 22^{\text {phox }}$ mutant stability, thereby permitting detection of $\mathrm{p} 22^{\text {phox }}$ in Cyba $a^{\text {nmf333 }}$-derived cells and tissue. Indeed, the $\mathrm{p} 22^{\text {phox }}$ protein was detected in peritoneal exudate cells, lamina propria, and the intestinal muscle layer of $C y b a^{n m f 333}$ mice in steady state and/or after upregulation by DSS-induced colitis (Fig. 2h, i). Thus, $\mathrm{H}_{2} \mathrm{O}_{2}$ production by Nox4 will persist in CGD patients with the CYBA p. 


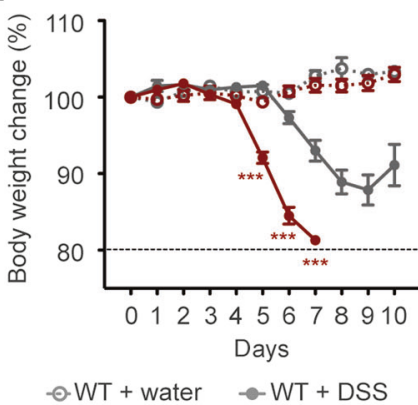

d

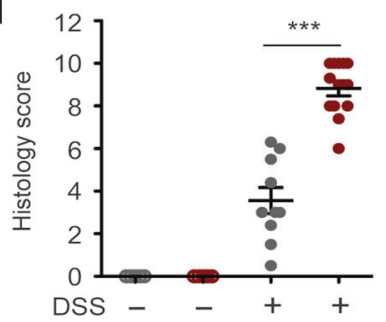

g

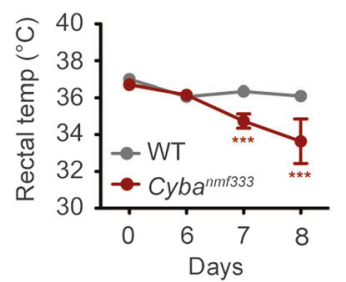

j

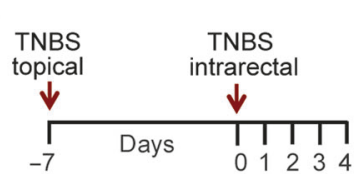

$\rightarrow$ WT + EtOH $\rightarrow$ WT + TNBS

$\odot \mathrm{Cyba}^{n m+333}+\mathrm{EtOH} \rightarrow \mathrm{Cyba}^{n m+333}+\mathrm{TNBS}$

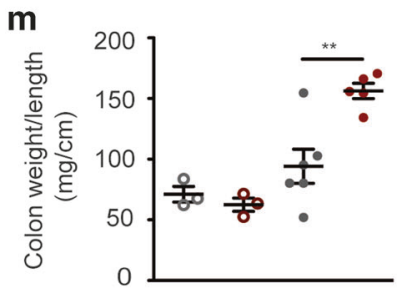

b

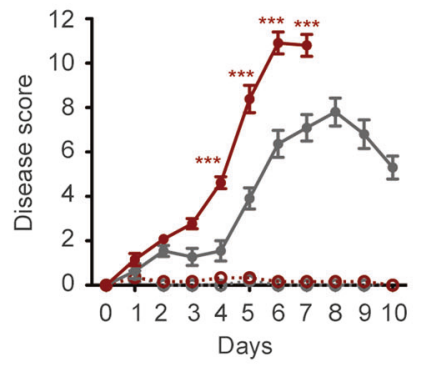

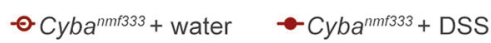

e

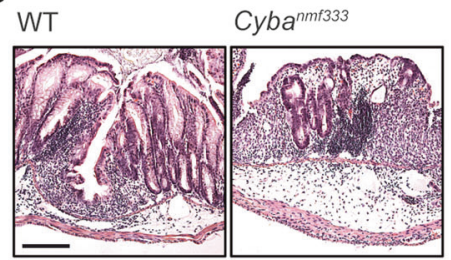

h

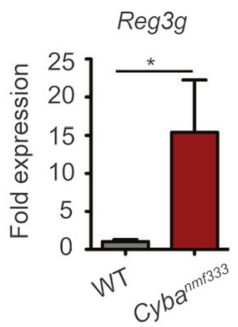

k

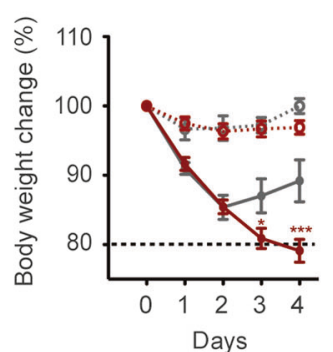

n

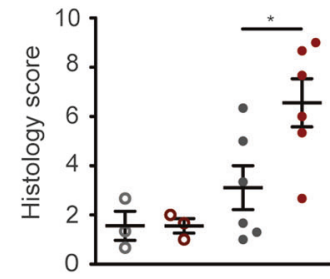

C

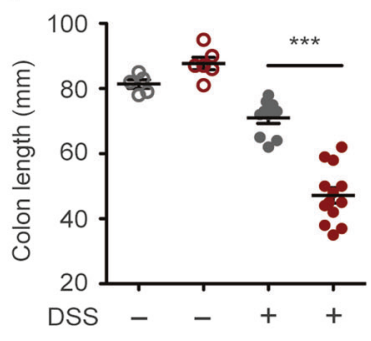

f

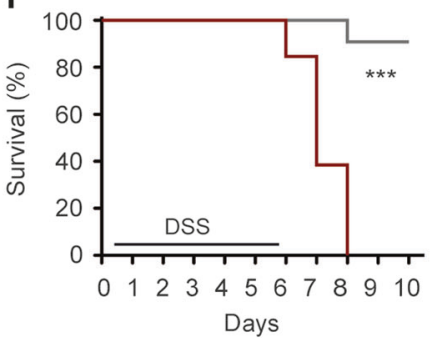

i

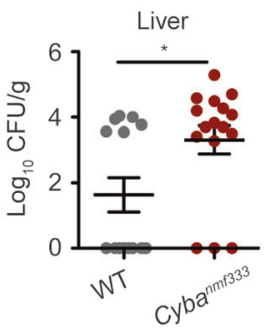

I

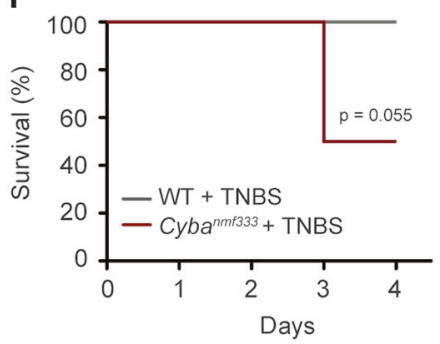

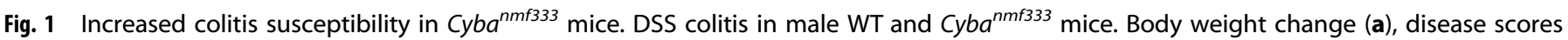
(b), colon length (c), histology scores (d), representative images of H\&E stained colon sections (e), and survival curve (f) of DSS-treated WT and Cyba $a^{n m 333}$ mice. Rectal temperature measured at days 0 and 6-8 (g), relative expression of colonic Reg3g mRNA at day 7 of DSS colitis (h), and bacterial translocation to the liver expressed as colony forming units (CFUs) at days 7 and 8 of DSS colitis (i). TNBS colitis in male WT and $C y b a^{n m f 333}$ mice. Scheme of TNBS model (j), body weight change (k), survival curve (I), colon weight/length ratio (m), and histology scores (n). Data are presented as mean \pm SEM of one $(\mathbf{h}, \mathbf{k}-\mathbf{n})$ or two $(\mathbf{a}-\mathbf{f}, \mathbf{g}, \mathbf{i})$ independent experiments. Each symbol represents an individual mouse; $n=5-6$ for control and $n=11-13$ for DSS groups (a-f); $n=16(\mathbf{g}, \mathbf{i}) ; n=6-7(\mathbf{h}) ; n=3$ for control and $n=6$ for TNBS groups (k-n). Data were analyzed using two-way ANOVA followed by Bonferroni post hoc test $(\mathbf{a}, \mathbf{b}, \mathbf{g}, \mathbf{k})$, Mantel-Cox test (f, I), or unpaired $t$-test $(\mathbf{c}, \mathbf{d}, \mathbf{h}, \mathbf{i}, \mathbf{m}, \mathbf{n})$. ${ }^{*} P<0.05,{ }^{* *} P<0.01$, and ${ }^{* *} P<0.001$ vs WT + DSS or TNBS. Scale bar $100 \mu \mathrm{m}$ 
a

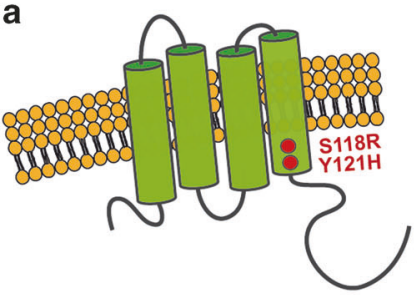

C

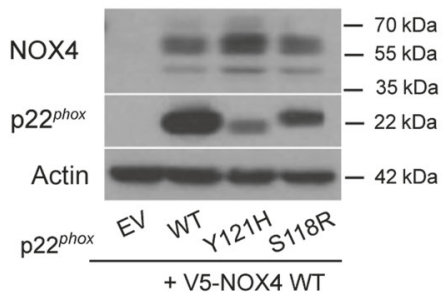

b

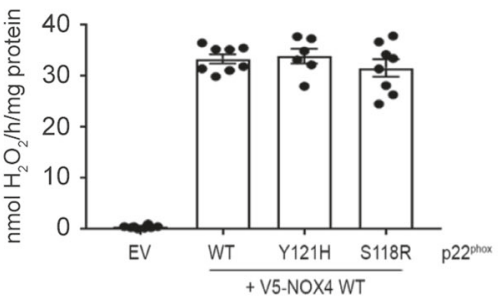

d

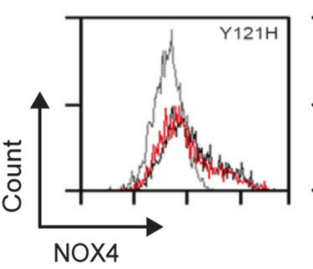

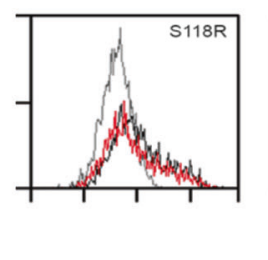

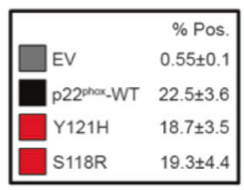

e

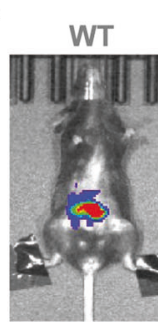

Cybb-1-

Nox1-1-

Cyba $a^{n m f 333}$

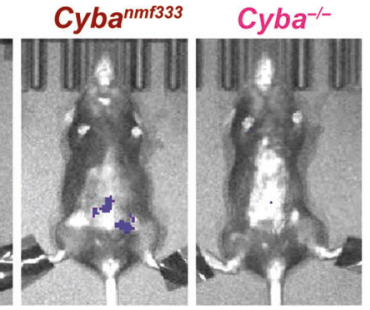

$\mathrm{p} / \mathrm{sec} / \mathrm{cm}^{2} / \mathrm{sr} \times 10^{\circ}$
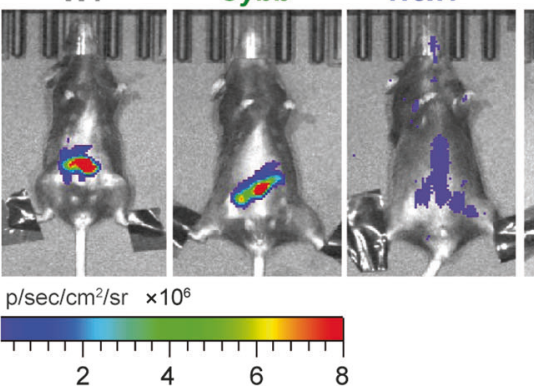

8

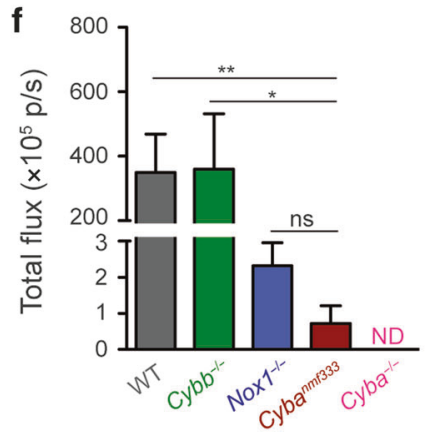

g

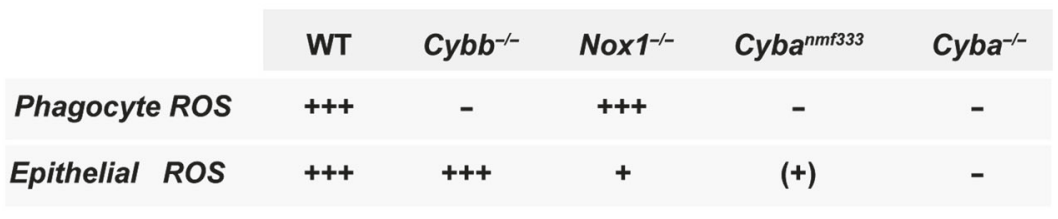

h

Peritoneal exudate cells

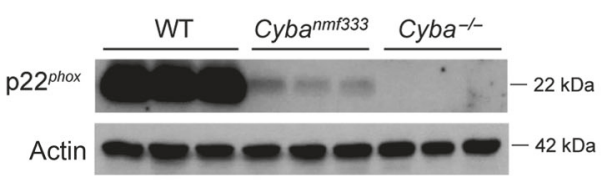

i

Lamina propria
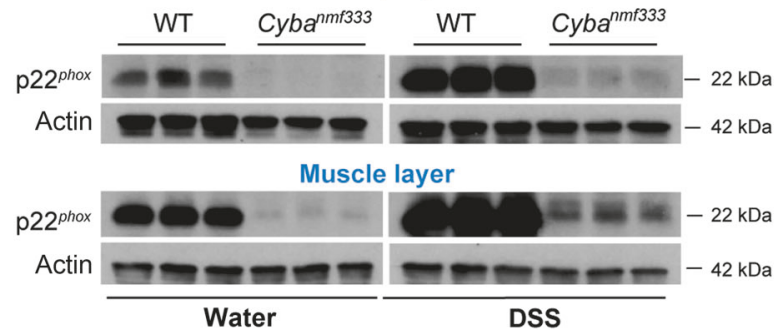

Fig. 2 NOX4 activity and residual ROS production in $C Y B A$ variant and $C y b a^{\text {nmf333 }}$ mice. Location of p22 $2^{\text {phox }} \mathrm{S} 118 \mathrm{R}$ and $\mathrm{Y}_{121} 1 \mathrm{H}$ mutants $(\mathbf{a}) . \mathrm{H}_{2} \mathrm{O}_{2}$ production (b), expression (c), and cell surface localization (d) of NOX4 co-expressed with p22 ${ }^{\text {phox }}$ WT or mutants in a cell-based assay (EV, empty vector). Comparison of in vivo ROS detection in female Cyba $a^{\text {nf } 333}$ (p22 $\left.{ }^{\text {phox }} \mathrm{Y} 121 \mathrm{H}\right)$, WT and indicated strains (e) and signal quantification (f). Nox-derived ROS by phagocytes or intestinal epithelial cells, deduced from Nox deletion/inactivation and in vivo detection,

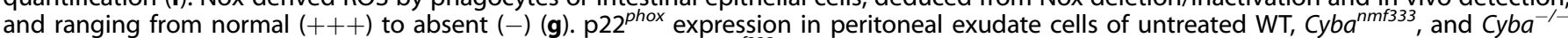
mice (h) and in lamina propria and muscle layer of WT and Cyba ${ }^{n m f 333}$ mice $+/-$ DSS treatment (i). Data are presented as mean \pm SEM of one $(\mathbf{h}, \mathbf{i})$ or three $(\mathbf{b}-\mathbf{f})$ independent experiments; $n=3-8(\mathbf{b}-\mathbf{d})$ and $n=3-10$ mice (f). Data were analyzed using one-way ANOVA followed by Dunn's multiple comparison test (f). ${ }^{*} P<0.05$ and ${ }^{* *} P<0.01$. ND not detectable, ns not significant

Ser118Arg variant, thereby setting the patient mutation apart from complete CYBA deletion.

Protective role of Nox4 in the Cyba $a^{\text {nmf333 }}$ colitis phenotype Although the overall response of $\mathrm{Nox}^{-1-}$ mice was comparable to WT mice in DSS colitis (Fig. S4), colonic Nox1 mRNA expression was moderately downregulated, while Nox4 mRNA showed significant upregulation in the acute colitis phase (Fig. 3a). This observation suggested that Nox4, a transcriptionally regulated and constitutively active oxidase, ${ }^{20}$ might be involved in the early stages of intestinal inflammation. To understand the functional implications for the Cyba $a^{\text {mff333 }}$ phenotype, we generated Cyba $a^{\text {nf } 333} /$ Nox $^{-1-}$ and Cyba ${ }^{\text {nmf333 }} /$ Nox $^{-1-}$ mice. Nox1 deletion in Cyba $a^{\text {nmf333 }}$ mice did not affect disease outcome when 

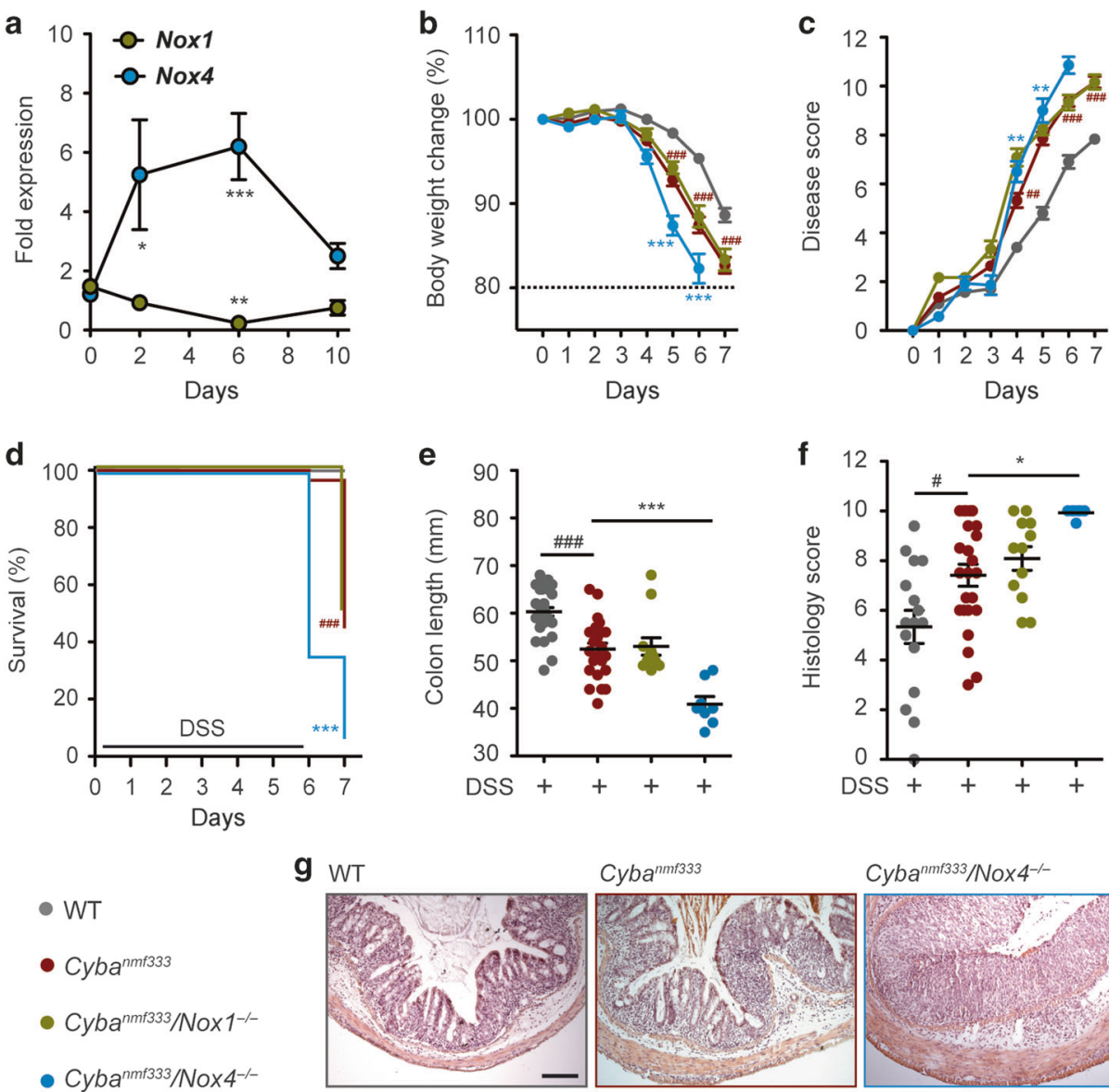

g WT
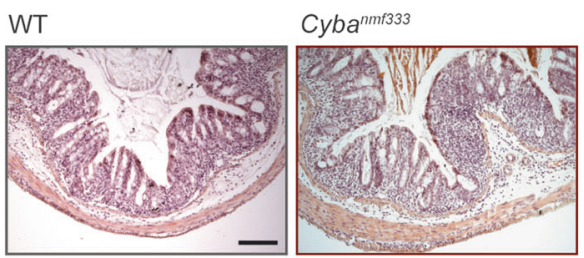

Cyba ${ }^{n m+333} / \mathrm{Nox}^{-/-}$

Fig. 3 Deletion of Nox4 aggravates colitis in Cyba $a^{n m 333}$ mice. Relative expression of colonic Nox 1 and Nox4 mRNA during DSS colitis in WT

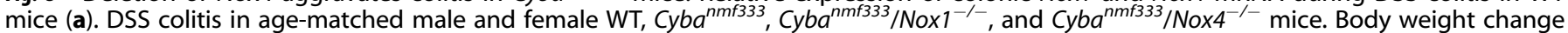
(b), disease scores (c), survival (d), colon length (e), histology scores (f), and representative images of H\&E stained colon sections (g) in DSStreated mice. Data are presented as mean \pm SEM of one (a) or two $(\mathbf{b}-\mathbf{g})$ independent experiments. Each symbol represents an individual mouse; $n=3-9$ mice (a); $n=12-30$ mice (b-f). Data were analyzed using one-way ANOVA followed by Dunn's multiple comparison test (a), two-way ANOVA followed by Bonferroni post hoc test $(\mathbf{b}, \mathbf{c})$, Mantel-Cox test (d), or one-way ANOVA followed by Bonferroni multiple comparison test $(\mathbf{e}, \mathbf{f}){ }^{*} P<0.05,{ }^{* *} P<0.01$, and ${ }^{* * *} P<0.001$ vs day 0 (a) or Cyba ${ }^{\text {nmf333 }}$ (b-f); ${ }^{\#} P<0.05$, ${ }^{\# \#} P<0.01$, and ${ }^{\# \# \#} P<0.001$ vs WT (b-f)

compared with Cyba $a^{\text {nmf333 }}$ mice (Fig. 3b-f), which was anticipated as the $\mathrm{p} 22^{\text {phox }} \mathrm{Y} 121 \mathrm{H}$ mutation did not support Nox1 activity in cell-based assays. ${ }^{18}$ In contrast, colitis severity was exacerbated in Cyba $a^{\text {nmf333 }} /$ Nox $^{-/-}$mice, with faster disease onset and earlier mortality (Fig. 3b-g). Thus, early upregulation of Nox4 during DSS colitis constitutes a protective mechanism, which is retained in vivo in the presence of the $n m f 333$ mutation as a functional Nox4 complex can be formed.

Bone marrow (BM) transplantation ameliorates colitis in Cyba $a^{n m f 333}$ mice

Aberrant mucosal cell infiltration in the colon of DSS-treated Cyba $a^{n f 333}$ mice (Fig. 1e) was characterized primarily by granulocyte transmigration, as shown by increased lamina propria $\mathrm{CD} 11 \mathrm{~b}^{+} \mathrm{Gr}-1^{+}$cell frequency (Fig. S5a, b), and the upregulation of the neutrophil chemokine $\mathrm{CxCl} 11$ (Fig. S5c). This prompted us to determine the net contribution of the $n m f 333$ mutation in the myeloid vs stromal compartment using BM-derived chimeric mice (Fig. S5d). The presence of WT p22 $2^{\text {phox }}$ in the hematopoietic cell compartment, i.e., presence of functional innate immune cell Nox2-derived superoxide, significantly improved colitis outcomes when compared with the presence of $\mathrm{p} 22^{\text {phox }} \mathrm{Y} 121 \mathrm{H}$ in the BM. Restoring immune cell superoxide reverted indicators of intestinal inflammation significantly, but not completely (Fig. S5e-i). Consistently, the presence of the $n m f 333$ mutation in BM rendered WT chimeric mice prone to severe colitis. These data indicate that ROS deficiency in the hematopoietic lineage is responsible for the bacterial dissemination and sepsis-like disease outcome, however other compartments, i.e., the intestinal epithelium also contribute to colitis susceptibility of Cyba $a^{n m f 33}$ mice.

The mucus barrier is compromised in $\mathrm{Cyb}^{\mathrm{nmf333}}$ mice

The intestinal epithelium provides the physical barrier to the gut environment, and this compartment was responsible for homeostatic ROS generation in WT mice (Fig. 2). While intestinal permeability was not affected (Fig. S3a), the colonic mucus layer showed substantial alterations in Cyba ${ }^{\text {nmf333 }}$ mice when compared with WT or single Nox deletion mice. Expression of the mucin Muc2 and the fucosyltransferase Fut 1 was comparable in WT and $C y b a^{\text {mff333 }}$ mice, while expression of Fut2, which catalyzes a1,2fucosylation of glycan chains, was decreased in mutant mice (Fig. 4a). FUT2 gene polymorphisms leading to ABH antigen nonsecretors have been associated with susceptibility to Crohn's disease. ${ }^{21}$ Muc2 distribution along the crypts was altered (Fig. 4b) and Ulex europaeus agglutinin 1 (UEA-1) staining, which detects Fuc-a-1-2-Gal linkages in fucosylated oligosaccharides, was slightly increased in Cyba ${ }^{\text {nmf } 333}$ mice (Fig. 4c). A trend toward neutral mucins may explain enhanced UEA-1 staining in Cyba $a^{n m f 33}$ mice. Morphometric analysis on semi-thin cross-sections from $C y b a^{n m f 333}$ mice indicated that the dense mucus layer was thinner 
a
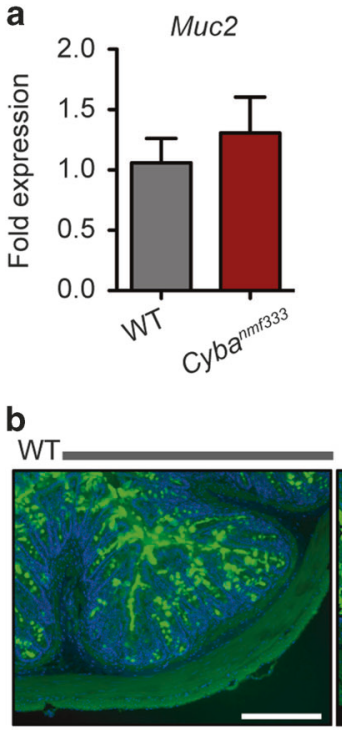

Muc2 DAPI

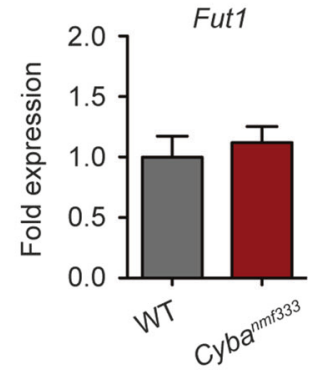

Cyba ${ }^{\text {nmf333 }}$

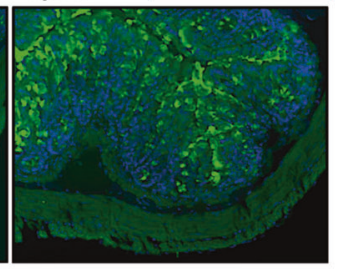

d
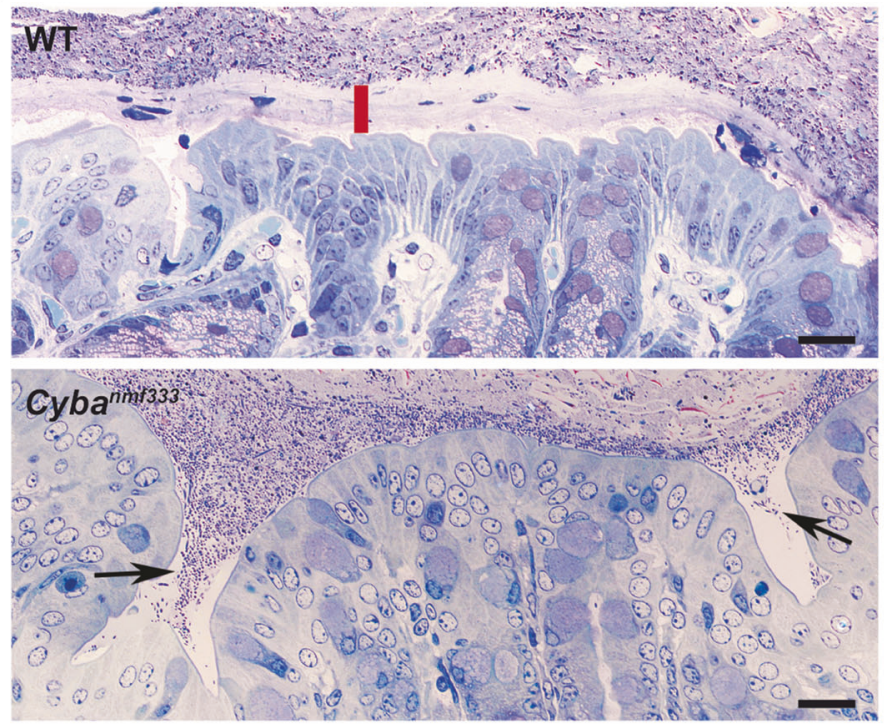
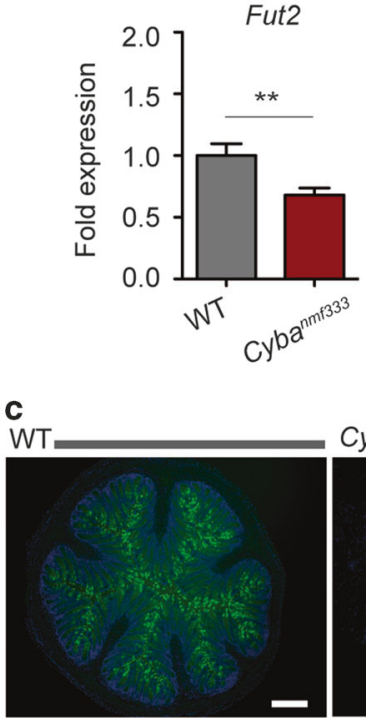

Cyba $a^{n m 333}$

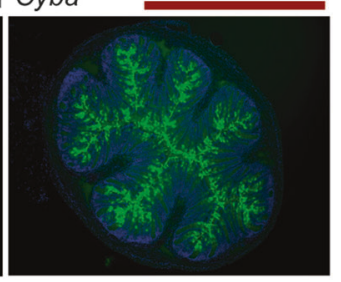

UEA-1 DAPI e

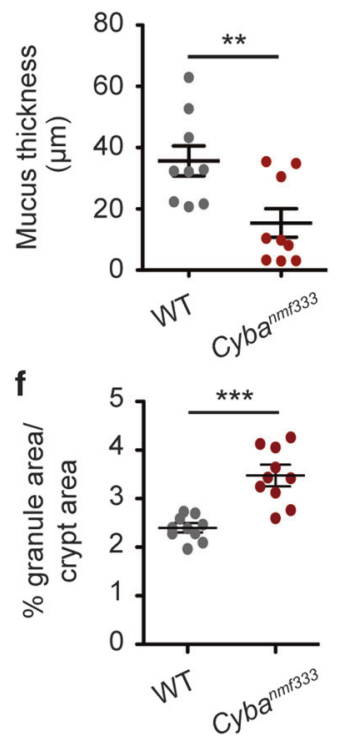

Fig. 4 Nox inactivation impairs the colonic mucus layer. Relative expression of colonic Muc2, Fut1, and Fut2 mRNA (a). Representative immunofluorescence images of fixed mouse colon probed with Muc2 (green), UEA-1 (green), and DAPI (blue) (b, c). Representative highresolution images of mouse colon (d) with dense mucus layer (red bar) and presence of bacteria inside crypts (arrows); mucus layer thickness (e) and goblet cell granule size (f) quantification. Data are presented as mean \pm SEM. Each symbol represents an individual mouse; $n=7-10$ mice $(\mathbf{a}) ; n=4-5$ mice $(\mathbf{b}, \mathbf{c}) ; n=9-10$ colon sections from three 15 -week-old mice for $\mathbf{d}$-f. Data were analyzed using unpaired $t$-test. ${ }^{* *} P<0.01$ and ${ }^{* * *} P<0.001$ vs WT. Scale bar $100 \mu \mathrm{m}(\mathbf{b}, \mathbf{c})$ and $20 \mu \mathrm{m}$ (d)

or even absent (Fig. 4d), permitting penetration of bacteria into the crypts. Quantification of mucus thickness and number of enlarged mucin granules per crypt further indicated that mucus secretion in Cyba ${ }^{\text {nmf333 }}$ mice was altered (Fig. 4e, f). Therefore, impaired ROS production by the Cyba $a^{\text {nmf } 333}$ mucosa induced changes in mucin secretion and distribution, enabling direct contact of the epithelium with intestinal microbiota. While this breach did not lead to spontaneous colitis in up to 12 months in our environmental conditions (data not shown), it likely accelerates microbe entry upon epithelial damage or metabolic changes, and overwhelms the ROS-deficient innate immune response. Supporting this notion, the absence of a severe colitis

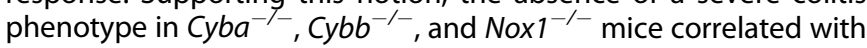
regular distribution of Muc2 in crypts (Fig. S6) and an undisrupted dense mucus layer (Fig. S7). In conclusion, epithelial redox alterations in the Cyba $a^{n m f 333}$ mouse are not functionally compensated by other eukaryotic or prokaryotic ROS sources, likely due to a certain ROS threshold being maintained by the genetic variant.

ROS deficiency leads to bacterial dysbiosis in Cyba $a^{n m f 333}$ mice The interactions of gut epithelium, mucus layer and the microbiota shape responses of these compartments. A key signal for reduced microbiota richness in IBD is an overall reduction in bacterial cell counts, ${ }^{22}$ and likewise the bacterial load was reduced in Cyba $a^{\text {nmf333 }}$ mice (Fig. 5a). Colonic content of WT and Cyba $a^{\text {nmf333 }}$ mice was analyzed by $16 \mathrm{~S}$ rRNA sequencing, as quantitative changes in $\mathrm{H}_{2} \mathrm{O}_{2}$ generation can alter bacterial community structure. ${ }^{12,23-25}$ No significant differences in diversity were 

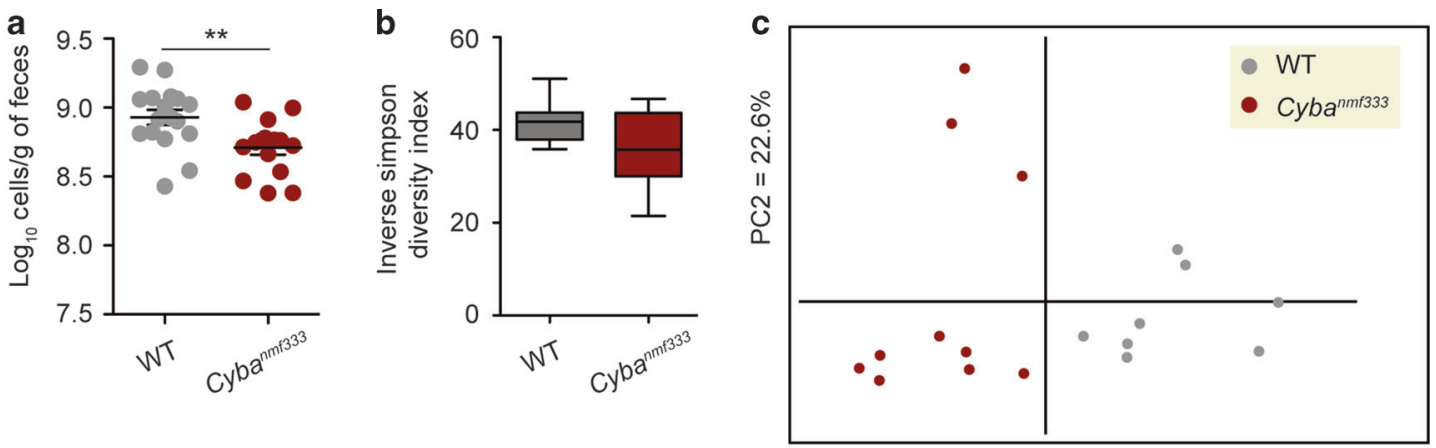

$\mathrm{PC} 1=23.6 \%$

d

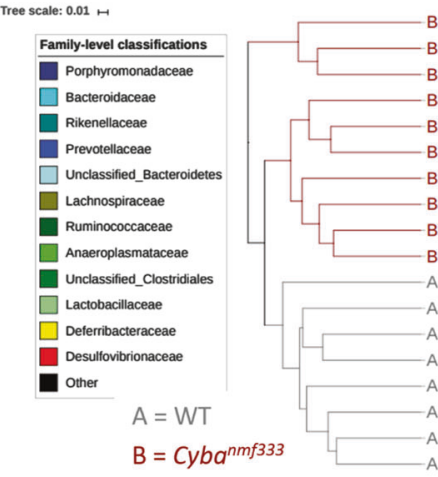

e Mucispirillum schaedleri

f

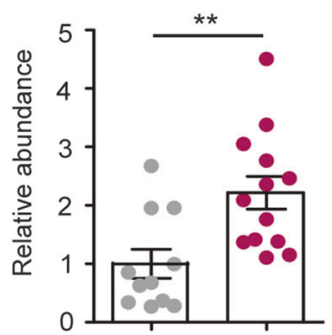

$\mathbf{h}$

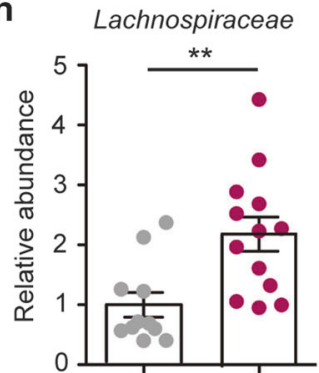

i Ruminococcaceae

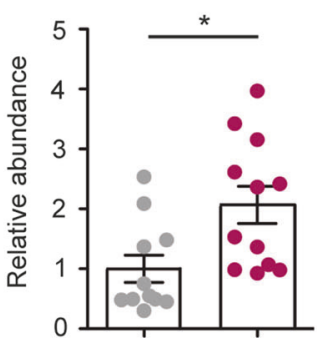

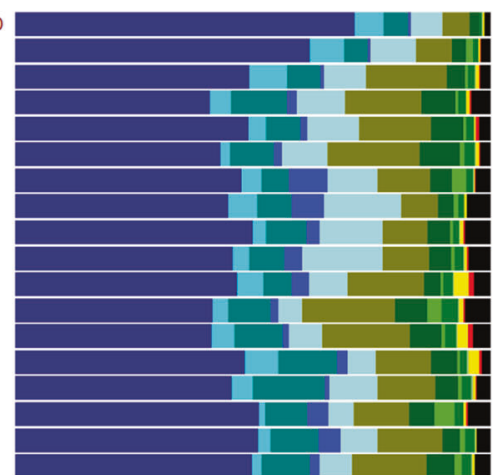

g Enterobacteriaceae

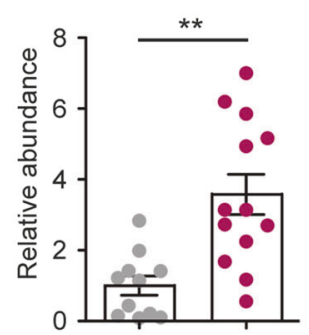

WT

Cyba $a^{n m f 333}$

Fig. 5 Dysbiotic microbiota in $C y b a^{n m f 333}$ mice. Bacterial load of untreated 8-9-week-old male and female WT and Cyba $a^{n m f 33}$ mice (a). $16 \mathrm{~S}$
rRNA gene sequencing of colonic content from 10-15-week-old male and female WT and Cyba $a^{n m f 33}$ mice $(n=8-10)$. Inverse Simpson Diversity index (b), principal component analysis (c), and Bray-Curtis dendrogram showing sample clustering by similarity, with proportional abundance at the family level between individual mouse samples across rows (d). Relative abundance of Mucispirillum schaedleri, proteobacteria, Enterobacteriaceae, Lachnospiraceae, and Ruminococcaceae determined by real time qPCR from the colonic content of 6-7week-old male and female WT and Cyba $a^{n m f 333}$ mice $(\mathbf{e}-\mathbf{i})$. Data are presented as mean \pm SEM of two independent experiments. Each symbol represents an individual mouse; $n=15-17$ mice $(\mathbf{a}) ; n=8-10$ (b-d); $n=11-13$ (e-i). Data were analyzed using unpaired (a, e-i) or Mann-Whitney (b) $t$-test. ${ }^{*} P<0.05$ and ${ }^{* *} P<0.01$ vs WT; $P=0.002$ and $P<0.001$ as assessed by the Parsimony and AMOVA tests respectively in mothur $(\mathbf{c}, \mathbf{d})$

observed when using the Inverse Simpson Diversity Index (Fig. 5b). However, principal component analyses (PCoA) and dendrograms based on Bray-Curtis dissimilarity index indicated distinct clustering of WT vs Cyba $a^{\text {nmf333 }}$ microbiota (Fig. 5c, d). Differences between WT and Cyba $a^{\text {nmf333 }}$ mouse microbiota were found at the
OTU level (Fig. S8, Table S1). Twelve OTUs with $>0.1 \%$ relative abundance were significantly different in proportional abundance between the two groups (metastats and LEfSe analysis; $P<0.05$ ). However, these OTUs corresponded to uncultured species, meaning the significance of these differences is unknown. These 
analyses were performed in randomized fashion, but on separately maintained mouse strains housed in the same room, as we previously observed that WT microbiota dominated over Noxdeficient microbiota in littermates. ${ }^{12}$ We further analyzed by qPCR selected phyla and species in colonic content collected from euthanized WT and Cyba $a^{\text {nmf333 }}$ mice, which were separated from littermates for one generation. We observed expansion of Mucispirillum schaedleri (10-20-fold), a mucus-associated pathobiont, ${ }^{26}$ and of proteobacteria including Enterobacteriaceae in Cyba ${ }^{\text {nmf333 }}$ mice, both putative markers of dysbiosis and inflammation (Fig. $5 \mathrm{e}-\mathrm{g}$ ). Abundance of actinobacteria or $E$. coli was not increased in Cyba $a^{n m f 33}$ mice, while Betaproteobacteria were reduced (Fig. S9a-c). Increased abundance of Lactobacillaceae, a signature of $\mathrm{Cyba}^{-1-}$ mice, was not detected in Cyba ${ }^{\text {nmf333 }}$ mice (Fig. S9d). Lachnospiraceae and Ruminococcaceae were more abundant in GPCR analysis, while not reaching significant difference when analyzed in separated strains (Fig. 5h, i, 16S rRNA results not shown). Lactobacilli as well as Delta/Gammaproteobacteria showed distinct gender-specific enrichment in both WT and Cyba $a^{n m f 333}$ mice (Fig. S9d, e). The dysbiotic microbiota in Cyba $a^{n m f 333}$ mice is likely associated with disease severity in DSS colitis, but it maintained, similar to WT microbiota, colonization resistance toward C. rodentium (Fig. S2).

Oral antibiotic treatment protects $C y b a^{n m f 333}$ mice from inflammation and mortality

Mucus and microbiota changes suggest that early barrier penetration and alterations in the commensal community in Cyba ${ }^{\text {nmf333 }}$ mice may function as initial triggers for an exacerbated response to gut microbiota, which is then compounded by the compromised antimicrobial defense in these mice. Thus, reducing the overall bacterial load and altering the microbiota composition by a non-absorbable, bactericidal antibiotic may ameliorate disease. Streptomycin was chosen as its impact on goblet cell function is minor while selectively removing facultative anaerobes and some anaerobes. ${ }^{27}$ We treated mice with streptomycin for 2 days prior to and during DSS challenge (Fig. 6a). Antibiotic treatment strongly reversed the $C y b a^{\text {mmf333 }}$ phenotype and achieved complete survival of mice (Fig. 6b-d). Colon tissues showed reduced signs of inflammation and diminished histological damage (Fig. 6e, f). This phenotype reversal was much stronger for Cyba $a^{\text {nmf333 }}$ mice than for WT mice. As expected, the streptomycin pretreatment reduced bacterial diversity in both Cyba $a^{\text {nf333 }}$ and WT mice (Fig. $6 \mathrm{~g}$ ) and modified the overall WT and Cyba $a^{\text {nmf333 }}$ microbiota as shown by PCoA and dendrograms (Fig. 6h, i). The higher proportional abundance of the proteobacteria phylum in fecal pellets collected at day -2 from Cyba ${ }^{\text {nmf333 }}$ mice was significantly reduced by streptomycin treatment (Fig. 6j). To determine whether the Cyba $a^{\text {nmf33 }}$ microbiota was per se colitogenic, we depleted the indigenous microbiota ${ }^{12}$ and transplanted WT or Cyba ${ }^{\text {nmf333 }}$ colonic/cecal matter into Cyba $a^{\text {nmf333 }}$ mice (Fig. S10a, b). Exposure to DSS revealed that WT microbiota did not rescue the Cyba $a^{n m f 333}$ colitis phenotype (Fig. S10c-g). Similarly, equal ratio co-housing of pups derived from separately maintained WT and Cyba $a^{\text {nmf33 }}$ mice did not alter the disease course or pathology in both mouse strains (Fig. S10h-m). This indicates that the colitis phenotype of Cybamf333 mice is predominantly influenced by the underlying genotype, which causes combined dysfunction in epithelial barrier homeostasis and innate immune responses, and effective treatments may include antibiotics that alter bacterial community structure.

\section{DISCUSSION}

Chronic Gl inflammation due to attenuated intestinal ROS production is not only a common manifestation in CGD, but is also associated with pediatric IBD and very early onset IBD (VEOIBD). Certain VEOIBD patients possess inactivating inherited or de novo variant alleles of innate immune NOX2 complex associated genes, or of epithelial NOX1 and DUOX2 genes. ${ }^{28-31}$ $C Y B A$ variants are considered rare, but the incidence is elevated in populations with high rates of consanguineous marriages ${ }^{32}$ and case reports note recurrent diarrhea and gastroenteritis in these patients. ${ }^{33,34}$ Modeling a CYBA mutational hotspot we show that the intestinal barrier of $C y b a^{n m f 333}$ mice is compromised in homeostasis, which predisposes to excessive inflammation and microbial dissemination when challenged or subjected to environmental influences. A similar defect might be present in certain CGD patients as elevated levels of antimicrobial antibodies have been detected even in the absence of colitis. ${ }^{35}$ Inactivating $C Y B A$ variants have wide-ranging effects, as this protein is required for the function of four NADPH oxidases (NOX1-4). Certain CYBA variants, for example introduction of an early stop codon or $\mathrm{p}$. Arg90GIn, will render NOX1-4 inactive, while other CYBA variants including p.Ser118Arg (shown here), p.Pro156Glu, and the common p.Tyr72His are selectively inactivating NOX1-3. ${ }^{18}$ Patients with selective CYBA variants will generate $\mathrm{H}_{2} \mathrm{O}_{2}$ via NOX4, and Cyba $a^{n m f 333}$ mice reflect this phenotype.

ROS generation is of such vital importance for physiological cell functions that deletion will trigger compensatory mechanisms in living organisms. For instance, we detected upregulation of Nox3 mRNA in the colon of Nox $1^{-1-}$ mice (Fig. S11a) and of Duox 2 in the colon of Cyba $a^{-1-}$ mice. ${ }^{12}$ In contrast, Cyba $a^{\text {nmf333 }}$ mice showed comparable expression of Duox2/Duoxa2 mRNA in homeostasis and during colitis (Fig. S11b, c). The diffusibility of $\mathrm{H}_{2} \mathrm{O}_{2}$ permits compensation of a missing ROS signal by other sources including bacteria such as Lactobacillus. ${ }^{12}$ Compensation by other ROS sources may explain why $\mathrm{Nox}^{-1-}$ and $\mathrm{Cyba}^{-1-}$ mice did not display any discernible colonic mucus defect, and did not show heightened susceptibility to DSS or bacterial colitis. As observed previously, Nox2 knockout mice recovered faster from DSS colitis, which is difficult to reconcile with the exaggerated colitis phenotypes reported for mice deficient in Nox2 complex components (Ncf1, Ncf4). ${ }^{2}$ Further, Duoxa ${ }^{-/-}$mice with combined Duox1/Duox2 inactivation did not differ from WT mice in DSS colitis. ${ }^{23}$ On the other hand, Nox1 and Duox2 seem to contribute to oxidative damage and spontaneous ileocolitis in mice with deficiency in the glutathione antioxidant system $\left(G p x 1^{-/-}\right.$ $\left.G p \times 2^{-1-}\right) .^{36,37}$ Beneficial compensatory mechanisms in humans and mice can be easily disrupted by environmental changes such as diet, ${ }^{12}$ infections, or drugs, thereby reinforcing low ROSinduced disease susceptibility. Continuously decreased ROS generation may permanently alter certain signaling responses while others stay intact, which may lead to transmitted epigenetic changes. ${ }^{38,39}$ This may explain why crossing Cyba $a^{\text {nm } 333}$ mice (Nox1-3 inactivated) with Nox4 $4^{-1-}$ mice did not result in the Cyba $a^{-/-}$phenotype. It is also conceivable that the $\mathrm{p} 22^{\text {phox }} \mathrm{Y} 121 \mathrm{H}$ mutation permits in some cell types minor formation of catalytically active Nox $1-3 / \mathrm{p} 22^{\text {phox }} \mathrm{Y} 121 \mathrm{H}$ heterodimers and consequently minor production of superoxide.

Key factors in promoting severe colitis in $C y b a^{n m f 333}$ mice were the compromised intestinal barrier and inefficient bacterial killing. Bacterial translocation into crypts due to a reduced or missing dense mucus layer was only observed in Cyba mutant mice, but not in $\mathrm{Cyba}^{-/-}, \mathrm{Cybb}^{-/-}$, or Nox $1^{-/-}$mice. This defect is clearly due to a loss in Nox generated superoxide production, which could not be substituted by Nox4- or Duox2-generated $\mathrm{H}_{2} \mathrm{O}_{2}$. In contrast to observations in $\mathrm{Cyba}^{-/-}$mice Lactobacillus-derived mucosal $\mathrm{H}_{2} \mathrm{O}_{2}$ could not compensate as the remaining $\mathrm{Cyba} a^{n m f 333}$ mucus did not support Lactobacillus overgrowth. The cell types expressing Nox4 in the colon and its cellular functions are still unknown, but Nox4 activity was upregulated and protective at early time points in DSS colitis in Cyba $a^{n m f 33}$ mice. The second factor leading to severe colitis in $\mathrm{Cyba}^{n \mathrm{mf333}}$ mice is the compromised immune cell response. Both, the repellant and antivirulence function of the host epithelium ${ }^{40}$ and the bacterial 
a

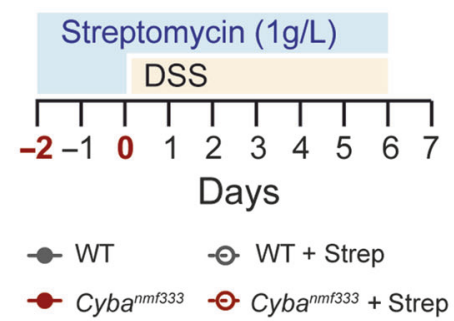

d

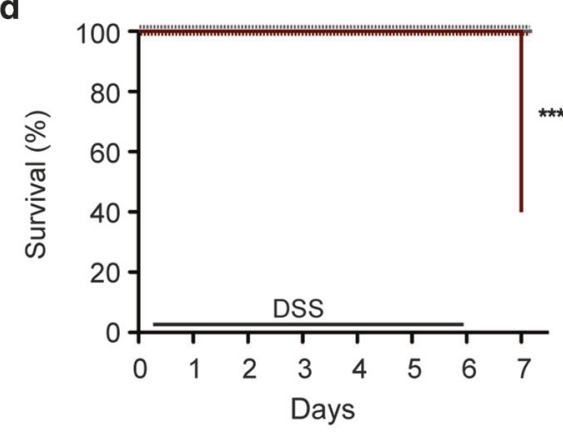

g
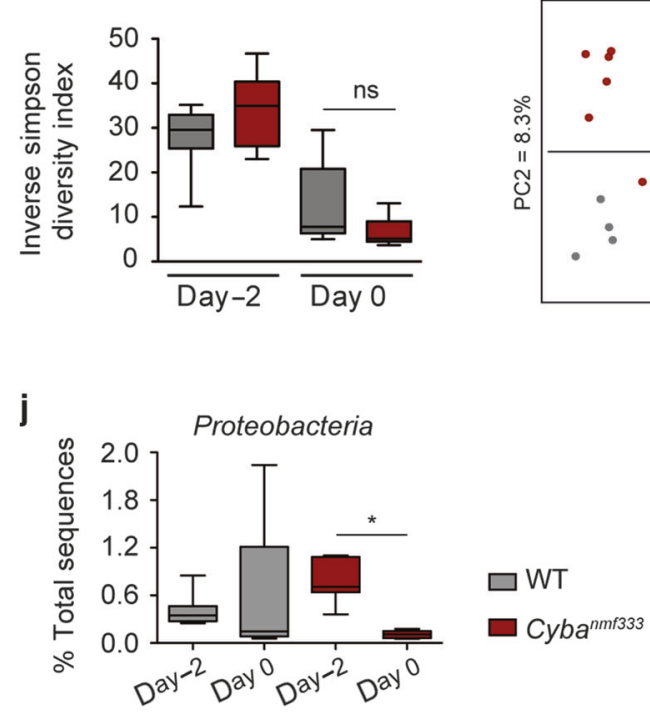

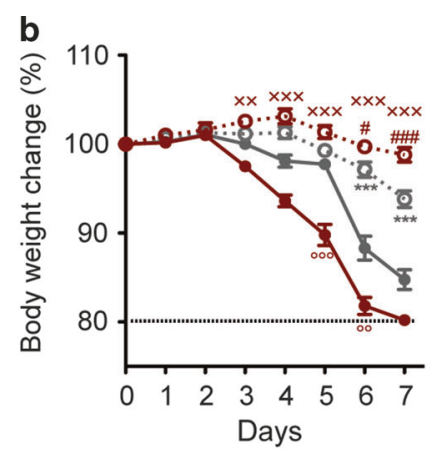

e

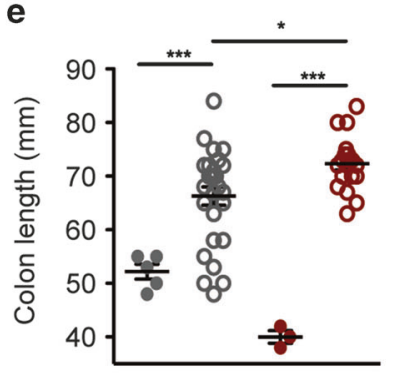

C

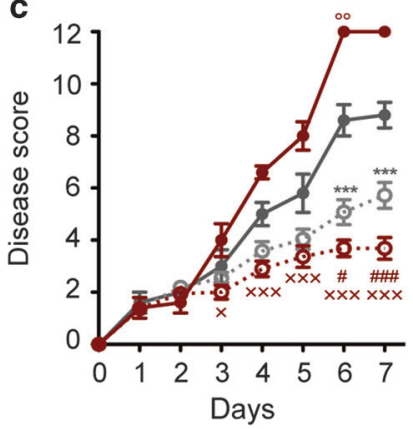

f

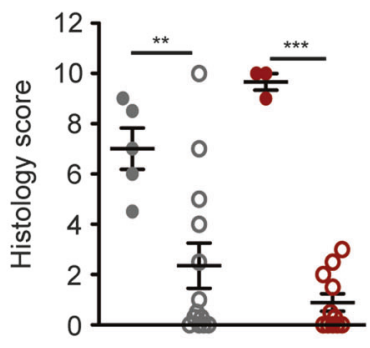

h

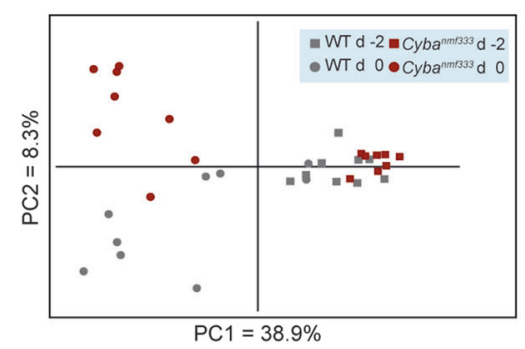

i

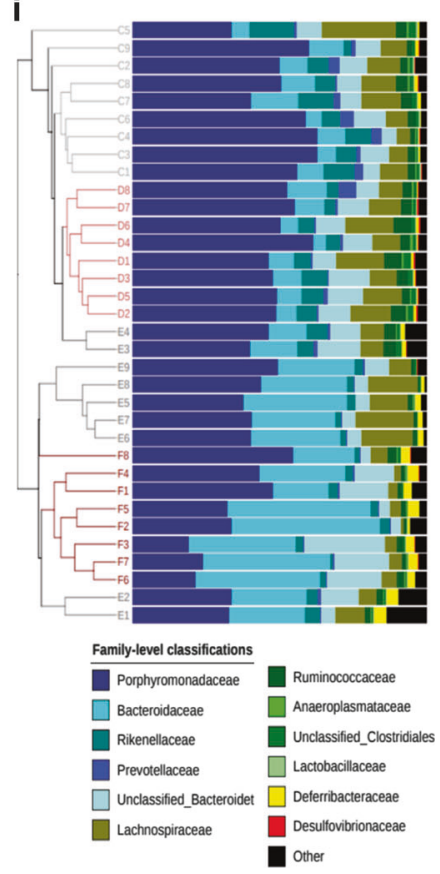

Fig. 6 Antibiotic treatment abolishes $C y b a^{n m f 333}$ susceptibility to colitis. Age-matched male and female WT and Cyba $a^{n m 333}$ mice were treated with streptomycin from day -2 to day 6 (a). Body weight change (b), disease scores (c), survival (d), colon length (e), and histology scores (f) of treated WT and Cyba $a^{\text {nff333 }}$ mice compared with untreated mice, all receiving DSS. 16S rRNA gene sequencing of fecal pellets collected at day -2 and day 0 from age-matched male and female mice. Inverse Simpson Diversity index (g), principal component analysis (h), and Bray-Curtis dendrogram showing sample clustering by similarity, with proportional abundance at the family level between mouse samples across rows (i). Proportional abundance of proteobacteria expressed as \% of total sequences before and after treatment (j). Data are presented as mean \pm SEM of three (a-f) or two $(\mathbf{g}-\mathbf{j})$ independent experiments. Each symbol represents an individual mouse; $n=19-27$ for streptomycin/DSS groups and $n=5$ for DSS only groups (b-f); $n=8-9$ mice $(\mathbf{g}-\mathbf{j})$. Data were analyzed using two-way ANOVA followed by Bonferroni post hoc test $(\mathbf{b}, \mathbf{c})$, Mantel-Cox test (d), one-way ANOVA followed by Bonferroni multiple comparison test $(\mathbf{e}, \mathbf{f}, \mathbf{j})$, or Mann-Whitney $t$-test $(\mathbf{g})$. ${ }^{*} P<0.05$, ${ }^{* *} P<0.01$, and ${ }^{* * *} P<0.001$ vs WT or relative control $(\mathbf{b}, \mathbf{c}, \mathbf{e}, \mathbf{f}, \mathbf{j}) ;{ }^{\circ}{ }^{\circ} P<0.01$ and ${ }^{\circ 0} P<0.001$ vs WT, ${ }^{\#} P<0.05$ and ${ }^{\# \# \#} P<0.001$ vs WT + Abx, ${ }^{\times} P<$ $0.05,{ }^{\times \times} P<0.01$, and ${ }^{\times \times \times} P<0.001$ vs $\operatorname{Cyba}^{n m f 333}(\mathbf{b}, \mathbf{c})$ 
killing mechanisms of innate immune cells ${ }^{41}$ are dependent on ROS generation for ongoing host protection. Reconstitution of myeloid ROS by allogeneic hematopoietic stem cell transplantation is curative for CGD patients including their GI manifestations and provided similarly an apparent benefit for $C y b a^{n m f 333}$ mice. Even more effective was antibiotic treatment. CGD patients are often placed on antimicrobial prophylaxis, and non-absorbable antibiotics were also beneficial in a cohort of pediatric IBD patients, ${ }^{42-44}$ suggesting that reducing certain phyla or the overall bacterial load might be a promising treatment.

In conclusion, the Cyba $a^{\text {nmf333 }}$ mouse represents a combined two-hit colitis model affecting the epithelial barrier and immune defense functions, both via attenuated ROS generation by a single gene variant. Recently, a VEOIBD patient with combined presence of hemizygous NOX1 p.Arg241Cys and homozygous CYBA p. Tyr72His was reported. ${ }^{45}$ The CYBA variant model presented here resembles this situation, as ROS levels will be attenuated in both compartments, while NOX4 function is preserved. ${ }^{18}$ A similar situation may also exist in patients with homozygous loss-offunction CYBC1 p.Tyr2Ter variants. Deficiency in CYBC1, an ERresident chaperone involved in NOX2-p22 $2^{\text {phox }}$ complex stabilization, triggers atypical CGD and is associated with IBD. ${ }^{46,47}$ It is not yet known if CYBC1 facilitates heterodimerization of NOX1, -3, -4 with $\mathrm{p} 22^{\text {phox }}$, but the function of some of these NOX enzymes may be selectively impaired. We postulate that CGD patients with inactivating variants affecting only the immune compartment or IBD patients with epithelial NOX1/DUOX2 variants have an additional genetic or environmental trigger for chronic colitis, albeit ROS deficiency will be the major IBD risk factor. ${ }^{10}$ Generating a comparable cohort of NADPH oxidase modified mouse strains for studies in homeostatic and colitis conditions permitted for the first time an environment-independent comparison. We demonstrate that an altered redox state due to mutant Cyba impinges on the gut barrier and host-microbiota interactions, which could be exploited for therapeutic strategies.

\section{METHODS}

Mice and colitis models

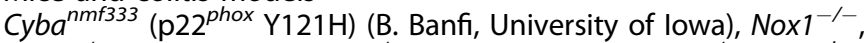
$\mathrm{Cybb}^{-/-}$(Nox2 KO), Nox4 $4^{-/-}$(Jackson Lab), Cyba ${ }^{-1-}$ (p22 $2^{\text {phox }}$ $\mathrm{KO})^{12}$ and newly created $\mathrm{Cyb} a^{\text {nmf333 }} / \mathrm{Nox}^{-1-}$ and Cyba ${ }^{\text {nmf333 }} /$ $\mathrm{Nox}^{-1-}$ mice were used. Mice were housed in individually ventilated cages for several years in the same room in a specific pathogen free facility. Strains were periodically backcrossed to inhouse bred C57BL/ 6 mice held in the same room. Mice were fed Teklad 2018 (Envigo) ad libitum and were supplied sterile water. Female and male mice were used in the following colitis models: (1) DSS colitis: after conditioning for 12 days 8-12-week-old, gender-matched male or female mice were subjected to $3 \%$ DSS in drinking water for 6 days, followed by water only. Daily scoring was for animal welfare, disease parameters, and body weight, and the disease activity index was calculated; ${ }^{48}$ (2) TNBS colitis: male mice $(30-35 \mathrm{~g})$ were pre-sensitized by topical application of $1 \%$ TNBS in acetone/olive oil $(4: 1 \mathrm{v} / \mathrm{v})$ on shaved dorsal skin, while control mice received vehicle only. After 7 days, mice were anaesthetized with $80 \mu \mathrm{l} / 10 \mathrm{~g}$ body weight of ketamine $(12 \mathrm{mg} /$ $\mathrm{ml}) /$ xylazine $(1.6 \mathrm{mg} / \mathrm{ml})$ and $2.5 \%$ TNBS in $50 \%$ ethanol/water $(100 \mu \mathrm{l})$ was administered intrarectally, while control mice received $100 \mu \mathrm{l}$ of $50 \%$ ethanol/water. Mice were observed daily as above; (3) Citrobacter rodentium colitis: 8-12-week-old female mice were infected by oral gavage with $0.3 \mathrm{ml}$ of $C$. rodentium $\left(\sim 1 \times 10^{9} \mathrm{CFU}\right)$ or growth medium only. Fecal pellets were collected at days 1,3 , and 7 post infection (dpi), while ceca and colons were collected at $7 \mathrm{dpi}$. Samples were homogenized in sterile PBS, and plated at serial dilutions onto MacConkey agar containing $35 \mu \mathrm{g} / \mathrm{ml}$ chloramphenicol, and CFU were determined after overnight incubation at $37^{\circ} \mathrm{C}$ in aerobic conditions. All animal experiments were performed in accordance with EU Directive 86/609/EEC, approved by the UCD Ethics Committee and authorized by the Irish Regulatory Authorities.

Measurement of ROS

In vivo ROS generation was determined using L-012 and IVIS imaging. ${ }^{49}$ Briefly, shaved mice were anesthetized with isoflurane and injected i.p. with $20 \mathrm{mg} / \mathrm{kg} \mathrm{L-012} \mathrm{dissolved} \mathrm{in} \mathrm{sterile} \mathrm{water.}$ The luminescent signal was detected by IVIS Spectrum and images were acquired $3 \mathrm{~min}$ after L-012 injection using Living Image ${ }^{\circledR}$ 4.5.2 software (PerkinElmer). Non-injected control mice were imaged for subtraction of the metabolic background. In vitro extracellular $\mathrm{H}_{2} \mathrm{O}_{2}$ production was measured using the homovanillic acid assay. ${ }^{20}$

\section{Histology and mucus assessment}

For histopathology distal colons were fixed in $10 \%$ buffered formalin. Deparaffinized and rehydrated $5 \mu \mathrm{m}$ sections were stained with H\&E. Colon pathology was scored in a blinded fashion. ${ }^{48}$ For Muc2 immunofluorescence rabbit polyclonal antiMuc2 antibody (Clone H-300, Santa Cruz Biotechnology) and goat anti-rabbit AlexaFluor-488 were used after fixation in Carnoy's solution. For high-resolution histology distal colons were fixed in $2.5 \%$ buffered glutaraldehyde. After post-fixation with $1 \%$ OsO4, samples were embedded into Epon, then $500 \mathrm{~nm}$ semi-thin crosssections were stained with toluidine blue, imaged using Nikon 80i microscope and analyzed with PlanApo $10 \times / 0.45$ and $100 \times / 1.4$. Full colon cross-section profiles were stitched together into digital slides by Photoshop, and mucus thickness was manually measured by FIJI. ${ }^{50}$ The area divided by the perimeter was defined as the average thickness of mucus. $N=3$ mice per genotype were analyzed (for each mouse three full cross-sections with the distance of $100 \mu \mathrm{m}$ in between each section). For goblet cell granule size quantification, high-resolution $10 \times$ panorama images $(n=$ 3-4 sections/mouse, $n=3$ mice/genotype) were used. Only fully developed mature crypts $(n=17-29)$ were analyzed. The granule sizes and the total area of crypts were measured using ImageJ and the average granule area/crypt area ratio was calculated.

Statistical analysis

Sample size $(n)$ denotes biological replicates and data points are presented as mean \pm standard error of the mean (SEM) of independent experiments as indicated. Statistical analysis was performed using GraphPad Prism for windows. Differences were considered significant when $P<0.05$.

\section{ACKNOWLEDGEMENTS}

The authors thank Sophie Shaw, CGEBM Aberdeen, for assistance with ENA submission. The work was supported by Science Foundation Ireland (U.G.K.), the National Children's Research Center (U.G.K. and B.B.), the European Crohn's and Colitis Organization (G.A.), the Medical Research Council MC/PC/15077 (G.A.) and MR/ P009824/1 (G.D.), and the Scottish Government's Rural and Environment Science and Analytical Services division (A.W.W.). The authors would also like to acknowledge the support of the Maxwell compute cluster funded by the University of Aberdeen.

\section{AUTHOR CONTRIBUTIONS}

Generation of data (G.A., A.K.S., S.O.N., E.C. and D.S.); analysis of data (G.A., A.K.S., S.O.N., E.C., D.S. and U.G.K.); manuscript preparation and statistical analysis (G.A., A.K.S., G.D.A., B.B., and U.G.K.); specialized equipment (W.G.); microbiota bioinformatics (A.W.W.); and study design (G.A. and U.G.K.), concept, and financial support (B.B. and U.G.K.).

\section{ADDITIONAL INFORMATION}

The online version of this article (https://doi.org/10.1038/s41385-019-0205-x) contains supplementary material, which is available to authorized users.

Competing interests: The authors declare no competing interests. 
Publisher's note Springer Nature remains neutral with regard to jurisdictional claims in published maps and institutional affiliations.

\section{REFERENCES}

1. Rada, B. \& Leto, T. L. Oxidative innate immune defenses by Nox/Duox family NADPH oxidases. Contrib. Microbiol. 15, 164-187 (2008).

2. Aviello, G. \& Knaus, U. G. NADPH oxidases and ROS signaling in the gastrointestinal tract. Mucosal Immunol. 11, 1011-1023 (2018).

3. Lambeth, J. D. \& Neish, A. S. Nox enzymes and new thinking on reactive oxygen: a double-edged sword revisited. Ann. Rev. Pathol. 9, 119-145 (2014)

4. Marks, D. J. et al. Inflammatory bowel disease in CGD reproduces the clinicopathological features of Crohn's disease. Am. J. Gastroenterol. 104, 117-124 (2009).

5. Barbato, M. et al. Chronic granulomatous disease mimicking early-onset Crohn's disease with cutaneous manifestations. BMC Pediatr. 14, 156 (2014).

6. Arimura, Y. et al. Intractable colitis associated with chronic granulomatous disease. J. Med. Microbiol. 55(Pt 11), 1587-1590 (2006).

7. Imanzade, F., Sayarri, A. \& Tajik, P. Ulcerative colitis associated with chronic granulomatous disease: case report. Gastroenterol. Hepatol. Bed Bench 8, 233-235 (2015).

8. Alimchandani, M. et al. Gastrointestinal histopathology in chronic granulomatous disease: a study of 87 patients. Am. J. Surg. Pathol. 37, 1365-1372 (2013).

9. Agarwal, S. \& Mayer, L. Diagnosis and treatment of gastrointestinal disorders in patients with primary immunodeficiency. Clin. Gastroenterol. Hepatol. 11, 1050-1063 (2013).

10. Huang, C. et al. Genetic risk for inflammatory bowel disease is a determinant of Crohn's disease development in chronic granulomatous disease. Inflamm. Bowel Dis. 22, 2794-2801 (2016).

11. Denson, L. A. et al. Clinical and genomic correlates of neutrophil reactive oxygen species production in pediatric patients with Crohn's disease. Gastroenterology 154, 2097-2110 (2018).

12. Pircalabioru, G. et al. Defensive mutualism rescues NADPH oxidase inactivation in gut infection. Cell Host Microbe 19, 651-663 (2016)

13. Nakano, Y. et al. Mutation of the Cyba gene encoding p22 causes vestibular and immune defects in mice. J. Clin. Invest. 118, 1176-1185 (2008).

14. Campbell, E. L. et al. Transmigrating neutrophils shape the mucosal microenvironment through localized oxygen depletion to influence resolution of inflammation. Immunity 40, 66-77 (2014).

15. Mullineaux-Sanders, C. et al. Citrobacter rodentium relies on commensals for colonization of the colonic mucosa. Cell Rep. 21, 3381-3389 (2017).

16. Lopez, C. A. et al. Virulence factors enhance Citrobacter rodentium expansion through aerobic respiration. Science 353, 1249-1253 (2016).

17. Stasia, M. J. CYBA encoding p22(phox), the cytochrome b558 alpha polypeptide: gene structure, expression, role and physiopathology. Gene 586, 27-35 (2016).

18. von Lohneysen, K., Noack, D., Jesaitis, A. J., Dinauer, M. C. \& Knaus, U. G. Mutational analysis reveals distinct features of the Nox4-p22 phox complex. J. Biol. Chem. 283, 35273-35282 (2008).

19. O'Neill, S. et al. Quantitative interaction analysis permits molecular insights into functional NOX4 NADPH oxidase heterodimer assembly. J. Biol. Chem. 293, 8750-8760 (2018).

20. Martyn, K. D., Frederick, L. M., von Loehneysen, K., Dinauer, M. C. \& Knaus, U. G. Functional analysis of Nox4 reveals unique characteristics compared to other NADPH oxidases. Cell Signal 18, 69-82 (2006).

21. McGovern, D. P. et al. Fucosyltransferase 2 (FUT2) non-secretor status is associated with Crohn's disease. Hum. Mol. Genet 19, 3468-3476 (2010).

22. Vandeputte, D. et al. Quantitative microbiome profiling links gut community variation to microbial load. Nature 551, 507-511 (2017).

23. Grasberger, H. et al. Increased expression of DUOX2 is an epithelial response to mucosal dysbiosis required for immune homeostasis in mouse intestine. Gastroenterology 149, 1849-1859 (2015).

24. Falcone, E. L. et al. Colitis susceptibility in $\mathrm{p} 47\left(\mathrm{phox}^{-/-}\right)$mice is mediated by the microbiome. Microbiome 4, 13 (2016).

25. Matziouridou, C. et al. iNOS- and NOX1-dependent ROS production maintains bacterial homeostasis in the ileum of mice. Mucosal Immunol. 11, 774-784 (2018).
26. Loy A., et al. Lifestyle and horizontal gene transfer-mediated evolution of Mucispirillum schaedleri, a core member of the murine gut microbiota. mSystems 2, e00171-16 (2017)

27. Wlodarska, M. et al. Antibiotic treatment alters the colonic mucus layer and predisposes the host to exacerbated Citrobacter rodentium-induced colitis. Infect. Immun. 79, 1536-1545 (2011).

28. Dhillon, S. S. et al. Variants in nicotinamide adenine dinucleotide phosphate oxidase complex components determine susceptibility to very early onset inflammatory bowel disease. Gastroenterology 147, 680-689 e682 (2014).

29. Hayes, P. et al. Defects in NADPH oxidase genes and in very early onset inflammatory bowel disease. Cell Mol. Gastroenterol. Hepatol. 1, 489-502 (2015).

30. Parlato, M. et al. First identification of biallelic inherited DUOX2 inactivating mutations as a cause of very early onset inflammatory bowel disease. Gastroenterology 153, 609-611 e603 (2017).

31. Schwerd, T. et al. NOX1 loss-of-function genetic variants in patients with inflammatory bowel disease. Mucosal Immunol. 11, 562-574 (2018).

32. Koker, M. Y. et al. Clinical, functional, and genetic characterization of chronic granulomatous disease in 89 Turkish patients. J. Allergy Clin. Immunol. 132, 1156-1163 e1155 (2013).

33. Koker, M. Y. et al. Six different CYBA mutations including three novel mutations in ten families from Turkey, resulting in autosomal recessive chronic granulomatous disease. Eur. J. Clin. Invest. 39, 311-319 (2009).

34. Teimourian, $\mathrm{S}$. et al. Characterization of six novel mutations in CYBA: the gene causing autosomal recessive chronic granulomatous disease. Br. J. Haematol. 141, 848-851 (2008).

35. Yu, J. E. et al. High levels of Crohn's disease-associated anti-microbial antibodies are present and independent of colitis in chronic granulomatous disease. Clin. Immunol. 138, 14-22 (2011).

36. Esworthy, R. S. et al. Nox1 causes ileocolitis in mice deficient in glutathione peroxidase-1 and -2. Free Radic. Biol. Med. 68, 315-325 (2014).

37. Chu, F. F. et al. Deficiency in Duox2 activity alleviates ileitis in GPx1- and GPx2knockout mice without affecting apoptosis incidence in the crypt epithelium. Redox Biol. 11, 144-156 (2017).

38. Mikhed, Y., Gorlach, A., Knaus, U. G. \& Daiber, A. Redox regulation of genome stability by effects on gene expression, epigenetic pathways and DNA damage/ repair. Redox Biol. 5, 275-289 (2015).

39. Cyr, A. R. \& Domann, F. E. The redox basis of epigenetic modifications: from mechanisms to functional consequences. Antioxid. Redox Signal 15, 551-589 (2011).

40. Alvarez, L. A. et al. NADPH oxidase-derived $\mathrm{H} 2 \mathrm{O} 2$ subverts pathogen signaling by oxidative phosphotyrosine conversion to PB-DOPA. Proc. Natl. Acad. Sci. USA 113, 10406-10411 (2016).

41. Winterbourn, C. C. \& Kettle, A. J. Redox reactions and microbial killing in the neutrophil phagosome. Antioxid. Redox Signal 18, 642-660 (2013).

42. Turner, D., Levine, A., Kolho, K. L., Shaoul, R. \& Ledder, O. Combination of oral antibiotics may be effective in severe pediatric ulcerative colitis: a preliminary report. J. Crohns Colitis 8, 1464-1470 (2014).

43. Lev-Tzion, R. et al. Oral vancomycin and gentamicin for treatment of very early onset inflammatory bowel disease. Digestion 95, 310-313 (2017).

44. Breton J., et al. Efficacy of combination antibiotic therapy for refractory pediatric inflammatory bowel disease. Inflamm. Bowel Dis. 25, 1586-1593 (2019).

45. Lipinski S., et al. Missense variants in NOX1 and p22phox in a case of very-earlyonset inflammatory bowel disease are functionally linked to NOD2. Cold Spring Harb. Mol Case Stud. 5, a002428 (2019).

46. Arnadottir, G. A. et al. A homozygous loss-of-function mutation leading to CYBC1 deficiency causes chronic granulomatous disease. Nat. Commun. 9, 4447 (2018).

47. Dinauer, M. C. Inflammatory consequences of inherited disorders affecting neutrophil function. Blood 133, 2130-2139 (2019).

48. Aviello, G., Corr, S. C., Johnston, D. G., O'Neill, L. A. \& Fallon, P. G. MyD88 adaptorlike (Mal) regulates intestinal homeostasis and colitis-associated colorectal cancer in mice. Am. J. Physiol. Gastrointest. Liver Physiol. 306, G769-G778 (2014).

49. Conroy, E. \& Aviello, G. Imaging intestinal ROS in homeostatic conditions using L012. Methods Mol. Biol. 1982, 313-327 (2019).

50. Schindelin, J. et al. Fiji: an open-source platform for biological-image analysis. Nat. Methods 9, 676-682 (2012). 